\title{
Universiteit
}

Leiden

The Netherlands

\section{A round up on some of the latest in the chemistry and biology of carbohydrates and carbohydrate-processing enzymes}

Vocadlo, D.; Overkleeft, H.S.

\section{Citation}

Vocadlo, D., \& Overkleeft, H. S. (2019). A round up on some of the latest in the chemistry and biology of carbohydrates and carbohydrate-processing enzymes. Current Opinion In Chemical Biology, 53(2019), A1-A3. doi:10.1016/j.cbpa.2019.11.010

Version: $\quad$ Publisher's Version

License: $\quad$ Licensed under Article 25fa Copyright Act/Law (Amendment Taverne)

Downloaded from: https://hdl.handle.net/1887/3200443

Note: To cite this publication please use the final published version (if applicable). 


\section{A round up on some of the latest in the chemistry and biology of carbohydrates and carbohydrate- processing enzymes David Vocadlo and Herman Overkleeft}

\author{
Current Opinion in Chemical Biology \\ 2019, 53:A1-A3 \\ This review comes from a themed issue on \\ Mechanistic Biology \\ Edited by Hermen S. Overkleeft and David J. \\ Vocadlo \\ https://doi.org/10.1016/j.cbpa.2019.11.010 \\ 1367-5931/@ 2019 Published by Elsevier Ltd.
}

\section{David Vocadlo}

Department of Chemistry, Simon Fraser University, Burnaby, British Columbia, V5A 1S6, Canada

Department of Molecular Biology and Biochemistry, Simon Fraser University, Burnaby, British Columbia, V5A 1S6, Canada e-mail:dvocadlo@sfu.ca

David Vocadlo joined the Department of Chemistry at Simon Fraser University in 2004. He received his $\mathrm{PhD}$ (2002) at the University of British Columbia with S.G. Withers and was a postdoctoral fellow (2003) at the University of California at Berkeley with C.R. Bertozzi. His interests center on developing and using chemical tools to further our understanding of the cellular and organismal roles of protein glycosylation. David Vocadlo is a Canada Research Chair in Chemical Glycobiology and Co-Director of the Centre for High-Throughput Chemical Biology.

\section{Herman Overkleeft}

Leiden Institute of Chemistry, Leiden University, Einsteinweg 55, 2300, RA Leiden, the Netherlands

e-mail: h.s.overkleeft@chem.leidenuniv.n|

Hermen Overkleeft studied chemistry at the University of Amsterdam, where he obtained his $\mathrm{PhD}$ degree in 1997 on his thesis entitled 'Azasugars: Synthesis and evaluation as glycosidase inhibitors'. He did postdoctoral research at Leiden University (1997-1999) and at the Harvard Medical School (1999-2001). Since 2001, he is a professor of Bioorganic Chemistry at Leiden University. His current research interests focus on the design and synthesis of inhibitors for glycosidases, glycosyl transferases, and proteases.
Carbohydrates are ubiquitous throughout nature, from bacteria to humans, and play diverse roles from serving as important structures that confer essential material properties to plants right on through to acting as signals that regulate intercellular and intracellular signaling within mammals. As chemists and biologists delve deeper into examining the structures of glycans and understanding the two superfamilies of enzymes that regulate these structures, it is becoming increasingly clear that the glycosyltransferases (GTs) that build up these structures and the glycoside hydrolases (GHs) that break them down are important tools for synthetic biology. Furthermore, both these classes of enzymes, as well as the carbohydrate recognition modules that recognize particular glycan epitopes, are emerging as therapeutic targets. Accordingly, researchers are pressing onward, developing new chemical biology tools that can be used to characterizing these important proteins, as well as working to elucidate their functional roles, while also refining existing tools to render them more widely useful for nonspecialists. The net result is that various branches of glycoscience are becoming more accessible to those outside the traditional aegis of this field, which is reflected by the increasing impact of glycoscience on biology, biotechnology, and clinical research.

In this issue of the Current Opinion in Chemical Biology, a diverse group of authors cover a swath of topics relevant to the area of chemical biology of carbohydrates, which have seen some recent notable advances. These topics, unsurprisingly, reflect the diversity of the field of glycoscience and span right from the fundamental physical organic reactivity of carbohydrates to bioinformatic analysis of the continuously expanding superfamily of GHs and through to the exploitation of chemical tools to create immune modulators with potential therapeutic benefits.

Starting in the domain of bioinformatics, Garron and Henrissat (https:// www.sciencedirect.com/science/article/pii/S1367593119300407) summarize the state of the exceptionally informative CAZy classification system in 2019, revealing how the discovery of new families of GHs continues unabated, essentially showing a sustained linear growth. The everincreasing amount of sequencing data is, however, leading to an 
exponential growth in the numbers of GHs and furnishing many more members of most $\mathrm{GH}$ families. This dramatic increase in sequence data, coupled with constant improvements in protein modeling will likely drive innovations in protein engineering of this important superfamily of enzymes. Furthermore, as noted by the authors, given the speed of sequencing efforts, it is almost certain that new families of GH enzymes will continue to be identified.

The glycosyl transfer reaction is a superficially simple reaction catalyzed by both GHs and GTs, yet nature exploits the ability to subtly vary the outcome to generate desired outcomes. Columbo and Bennet (https://www.sciencedirect.com/science/article/pii/ S1367593119300699) cover the physical organic chemistry of glycosyl transfer reactions in both solution and enzyme active sites. The latest advances in understanding the nature of the high energy intermediates and transition states of these complex reactions are summarized, offering new insights into how these reactions might be controlled by researchers to deliver products with desired stereochemical outcomes.

The engineering of protein glycosylation has long been recognized as having a major influence on the pharmacokinetic properties of proteins and, more recently, as influencing their effects of the immune system. Creating homogenous glycoproteins remains a major challenge, particularly using only chemical synthetic methods. The use of wild-type enzymes and their engineered counterparts has, in recent years, opened up new possibilities for biosynthesis. Fairbanks (https:// www.sciencedirect.com/science/article/pii/

S1367593118301790) summarizes the latest developments on the enzymatic synthesis of homogenous glycoproteins, with a particular focus on the use of various endohexosaminidases for creating specific $\mathrm{N}$ glycan structures on proteins.

The constantly growing set of carbohydrate recognition modules mediate both physiological and pathophysiological processes right from viruses to mammals. Bacteria, for example, use cell surface glycans to drive invasion and colonization of host tissues. Meiers et al (https://www.sciencedirect.com/science/article/pii/ S1367593119300079) describe advances in lectin antagonists, illustrating the state of the field using examples from bacterial, viral, and mammalian lectins. This area continues to gain interest as new lectins are uncovered and antagonists start to see increasing utility both as research tools and in the clinic.

Decoding the connection between glycan epitopes and the proteins that bind them in a physiological context remains a challenge. Wu and Kohler (https://www. sciencedirect.com/science/article/pii/

S1367593119300109) detail the latest in creating chemically modified carbohydrates and glycoconjugates that contain strategically positioned photoreactive moieties. These photoaffinity ligands can be used directly or as metabolic precursors to enable photocrosslinking to carbohydrate receptors either in lysates or in cellular models.

In nature, the greatest diversity in glycan structures is unsurprisingly found among bacteria, which must adapt to occupy a dizzyingly diverse set of environmental niches. Imperiali (https://www.sciencedirect.com/ science/article/pii/S1367593119300596) discusses how recent developments in analytical methods continue to unearth new monosaccharides and glycan structures within bacteria. Furthermore, these bacterial glycans also become diversified through a range of downstream modifications. The creation of new chemical tools ranging from arrays of bacterial glycans to advancing new metabolic labeling agents for these unusual bacterial monosaccharides are emerging as important strategies to uncover the roles of these structures in host-microbe interactions.

Continuing in the domain of prokaryotes, the discovery of N-linked glycosylation within bacteria opened up an active area of synthetic biology focused on glycoengineering of proteins for a range of uses including creating vaccines. Nothaft and Szymanski (https://www.sciencedirect. com/science/article/pii/S1367593119300572) offer up a summary of the latest advances in N-linked glycosylation within bacteria, highlighting the increasing diversity of $\mathrm{N}$-glycosylation systems that have been identified while also noting how glycosylation is now starting to be found on other animo acids aside from asparagine. The authors also touch on the structure and function of these newly emerging enzymes, as well as consider prospects for their exploitation in glycoengineering.

The enzymes that polymerize the essential peptidoglycan cell wall of bacteria are established antibiotic targets. The peptidoglycan crosslinking mediated by penicillin binding proteins accommodate a range of amino acids. Some penicillin binding proteins can also catalyze exchange with a single D-amino acid, and Taguchi, Kahne, and Walker (https://www.sciencedirect. com/science/article/pii/S1367593119300778) summarize recent advances in metabolic labeling of the peptidoglycan with functionalized or fluorescently labeled D-amino acids. These, and other, strategies are permitting new insights into the peptidoglycan and uncovering continuing twists in the regulation, assembly, and remodeling of this important carbohydrate polymer.

Lipopolysaccharides are important structural and functional components within the inner membrane of gramnegative bacteria. The biosynthesis and transport to 
these inner membranes constitute attractive starting points for antibiotics discovery, also considering that antibiotics effective against gram-negative bacteria are scarce. Sweeney and Lowary (https://www.sciencedirect. com/science/article/pii/S1367593119300055) summarize the latest in molecular insights into how bacterial lipopolysaccharides are assembled and exported. They focus on some highly conserved, and therefore highly attractive from a drug discovery perspective, processes controlling lipopolysaccharide chain length during biosynthesis and how this affects their transport.

Turning to metazoans, the mechanisms by which the OGlcNAc modification of nuclear and cytoplasmic enzymes contribute to cellular homeostasis has emerged as a major topic of interest. Remarkably, only two enzymes regulate levels of this modification on hundreds of proteins. King et al (https://www.sciencedirect.com/ science/article/pii/S1367593119300663) focus on the molecular mechanisms by which O-GlcNAc transferase and its antagonistically acting partner O-GlcNAcase are themselves regulated. The authors integrate structural insights into these two processing enzymes along with molecular studies providing a critical assessment of a set of complex regulatory processes.

Turning back to chemical glycobiology tools and techniques, Wu et al (https://www.sciencedirect.com/ science/article/pii/S1367593119300626) review the latest developments in the design of covalent, mechanism-based, and irreversible GH inhibitors and their application as activity-based probes in the domains of biomedicine and biotechnology. The overview shows that activity-based protein profiling strategies can now reliably be implemented both for the discovery of new retaining GHs and for the discovery of inhibitors for these but that bringing the technology to bear toward inverting GHs and GTs requires further developments.
Continuing in the domain of eukaryotes, Aerts et al (https://www.sciencedirect.com/science/article/pii/

S1367593119300869) summarize the development of chemical biology tools for the enzyme $\beta$-glucocerebrosidase, deficiency of which was first identified as causing the lysosomal storage disease known as Gaucher disease. This enzyme has become of increasing interest and the focus of translational efforts ever since being uncovered as the greatest genetic risk factor for Parkinson disease. The creation of new chemical tools ranging from isotope-coded substrates and standards for mass spectrometry to activity-based proteomics probes will doubtless start to have major impacts in improving our understanding of the biology of glucosylsphingosine and $\beta$-glucocerebrosidase itself - hopefully enabling the creation of sorely needed new and improved therapies for patients with Gaucher disease and Parkinson disease.

In summary, the creation of new chemical biology tools and methods continue to be - as nicely illustrated by this set of articles - integral to the advancement of glycoscience. We expect that the set of review articles presented here should serve to update readers in these selected areas, while also serving as a source for inspiration to those working in the many branches of the widening canopy of glycoscience as its impacts continue to expand and reach into adjacent fields of research.

\section{Funding}

This work was supported by grants from the Canadian Institutes of Health Research (CIHR) (DJV; PJT148732, PJT-156202), the Natural Sciences and Engineering Council of Canada (Discovery-RGPIN298406), and the Netherlands Organization for Scientific Research (NWO; TOP grant to HSO); and the European Research Council (ERG-2011-AdG-290836 'Chembiosphing' to HSO). DJV is a Tier I Canada Research Chair in Chemical Biology. 